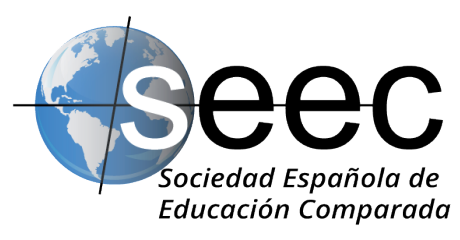

\title{
El Espacio Europeo de Educación Superior y su sello de calidad
}

The European Higher Education
Area and its quality seal

\section{María Matarranz García*}

DOI: $10.5944 /$ reec.37.2021.27728

Recibido: 17 de junio de 2020 Aceptado: 12 de noviembre de 2020

*María Matarranz García: Doctora en Educación por la Universidad Autónoma de Madrid. Profesora en la Facultad de Ciencias de la Salud y de la Educación en la Universidad a Distancia de Madrid (UDIMA). Miembro del Grupo de Investigación sobre «Políticas Educativas Supranacionales». Subdirectora de la Journal of Supranational Policies of Education. Datos de contacto: e-mail: maria.matarranz@udima.es 


\title{
Resumen
}

Desde la Declaración de la Sorbona en el año 1999 hasta la actualidad han pasado dos décadas, periodo de tiempo en el que hemos sido testigos de los grandes cambios que se han producido en los sistemas de educación superior en muchos países del mundo, en concreto los países pertenecientes al Espacio Europeo de Educación Superior (EEES).

Comenzaron siendo cuatro países los que firmaron la Declaración de 1999, hoy son ya cuarenta y ocho los países implicados en el EEES.

En el presente artículo se realiza un recorrido por los hitos que han ido configurando y amasando este Espacio. Abordaremos, en primer lugar, las cuestiones más relevantes tratadas en los distintos encuentros de los ministros de educación superior. Seguidamente, nos detendremos en uno de los indicadores más relevantes del EEES: los sistemas de garantía de calidad que, fruto del Proceso de Bolonia, se han desplegado tanto a nivel supranacional como a nivel nacional. Realizaremos una panorámica de la implantación de la calidad educativa en los países. Finalmente, reflexionaremos sobre el impacto que la perspectiva de la calidad educativa ha tenido en los países del Espacio Europeo de Educación Superior.

Palabras clave: Espacio Europeo de Educación Superior; Política Educativa Supranacional; Política Educativa; Calidad Educativa.

\begin{abstract}
Two decades have passed from the Sorbonne Declaration in 1999 to the present day, a period of time in which we have witnessed the great changes that have occurred in higher education systems in many countries of the world, specifically the countries belonging to the European Higher Education Area (EHEA).

Four countries started by signing the 1999 Declaration, today there are already fortyeight countries involved in the EHEA.

In this article, a tour of the milestones that have been shaping and kneading the EHEA is made, addressing the most relevant issues addressed in the different meetings of the ministers of higher education. Next, we will stop at one of the most relevant indicators of the EHEA: the quality assurance systems that, because of the Bologna Process, have been deployed both at the supranational and national levels. We will make an overview of the implementation of educational quality in the countries. Finally, we will reflect on the impact that the perspective of educational quality has had in the countries of the European Higher Education Area.
\end{abstract}

Keywords: European Higher Education Area; Supranational Policies of Education; Educational Policy; Educational Quality. 


\section{Introducción}

A comienzos del siglo XXI nos encontramos con dos procesos importantes que buscan la armonización en materia educativa de los Estados miembros: el Proceso de Copenhague (2002) y el Proceso de Bolonia (1999), dirigidos a la Formación Profesional y la Educación Superior, respectivamente. Ambos procesos promueven la cooperación y la convergencia a escala europea en los sistemas de enseñanza en educación superior.

Lejos queda ya aquel primer encuentro en Sorbona que inspiró lo que hoy conocemos como el Espacio Europeo de Educación Superior.

«Recientemente, el proceso europeo ha dado pasos de extrema importancia. A pesar de la relevancia que ello tiene, no deberíamos olvidar que al hablar de Europa no solo deberíamos referirnos al euro, los bancos y la economía, sino que también debemos pensar en una Europa de conocimientos. Es deber nuestro el consolidar y desarrollar las dimensiones intelectuales, culturales, sociales y técnicas de nuestro continente. Éstas han sido modeladas, en gran medida, por las universidades, quienes todavía desempeñan un papel imprescindible en su desarrollo». (Sorbona, 1998)

El nacimiento del Espacio Europeo de Educación Superior (EEES) con la declaración de la Sorbona de París el año 1998 dio el pistoletazo de salida a una de las iniciativas más emblemáticas y conocidas de la Unión, cuya finalidad última era hacer de Europa un espacio único para los estudiantes de educación superior, caracterizado por la excelencia en la docencia y en la investigación.

En dicho encuentro en París, los ministros de los Estados miembros decidieron trabajar en la «creación de una zona europea dedicada a la Educación Superior, donde las identidades nacionales y los intereses comunes puedan relacionarse y reforzarse para el beneficio de Europa, de sus estudiantes y en general de sus ciudadanos» (Declaración de la Sorbona, 1998, párrafo 14).

Esta participación que los ministros denominaron zona europea de la educación superior, supondría, para los países integrantes del EEES, una serie de reformas en sus instituciones educativas tanto a nivel estructural como a nivel curricular.

Los países participantes en este primer encuentro y que firmaron dicha declaración fueron Francia, Alemania, Italia y Reino Unido. Este pacto político prestaba una importancia nunca antes dada a la educación superior en Europa y cambiaría de manera determinante la organización y el funcionamiento de las instituciones de educación universitaria pertenecientes a la zona. Lo veremos detenidamente a continuación.

\section{El EEES: Veinte años de cooperación en Educación Superior}

\subsection{Los comienzos del Espacio Europeo de Educación Superior (1999-2010)}

Tras el encuentro en Sorbona (1998), hubo un segundo encuentro en 1999, en el que se firmó la Declaración de Bolonia, en ella los ministros de los países participantes se comprometieron a tratar de armonizar las políticas acerca de sus sistemas de educación universitaria por medio de los siguientes seis elementos (u objetivos) comunes: 
- La comprensividad y la comparabilidad entre las titulaciones, esto incluiría el Suplemento al título de educación superior que permitiría la descripción y definición detallada de cada uno de los títulos, facilitando al mismo tiempo el acceso de los egresados al mercado laboral.

- Una estructura de créditos en las titulaciones: la educación universitaria tendría una estructura común de dos niveles, el primer nivel correspondiente a los estudios de primer ciclo será el Grado, el segundo nivel correspondiente a los estudios de segundo ciclo será el Postgrado. El acceso al segundo ciclo o Posgrado solo podrá realizarse una vez se haya superado el primer ciclo o Grado.

- Un Sistema Europeo de Transferencia de Créditos (European Credit Transfer and Accumulation System, ECTS por sus siglas en inglés), que permitiría un sistema de créditos académicos comparable entre distintos países e instituciones de educación superior. Cada uno de los créditos que constituyen las titulaciones define el tiempo total de trabajo del estudiante.

- Promoción de la movilidad de estudiantes y profesores de educación superior.

- Titulaciones conjuntas en las que participen distintas instituciones de Educación Superior y el aseguramiento de la calidad de la educación en los países participantes.

- Promoción de la dimensión europea en la educación superior.

Tras la Declaración de Bolonia en 1999 han sido varios los encuentros entre ministros de educación superior y otros agentes implicados en el proceso de Bolonia, encuentros que han permitido la continuidad de este proyecto hasta la actualidad. Los ministros europeos en funciones en educación superior se reúnen periódicamente para estudiar los avances realizados y establecer direcciones y prioridades del proceso que la educación superior debe seguir.

En el año 2001 su reunión es en Praga, tras la cual se elabora un comunicado que recoge las cuestiones tratadas en ella titulado: «Fomentando el Proceso de Bolonia». En este encuentro los correspondientes ministros de educación superior revisan los objetivos planteados en la Declaración de Bolonia, así como su alcance hasta el momento. Dicha revisión la realizan sobre cada uno de los elementos que componen el EEES ya descritos: la adopción de un sistema fácilmente legible y comparable, la implantación de un sistema basado esencialmente en dos ciclos, el establecimiento de un sistema de créditos, la promoción de la movilidad de estudiantes y profesores, y la promoción de la cooperación europea en la garantía de calidad. Viendo los ministros un alto grado de alcance de dichos objetivos añaden elementos importantes tales como:

- Garantizar la calidad mediante mecanismos de evaluación, acreditación y certificación.

- Fomentar el aprendizaje a lo largo de la vida (Lifelong Learning).

- Fomentar la calidad de las instituciones y de los estudiantes de Educación Superior, considerando la calidad como la condición básica para la confianza, la relevancia, la movilidad, la compatibilidad y el atractivo del EEES. 
- Promocionar el interés por el Área de Educación Superior Europea, de forma que se generen redes de intercambio y colaboración con estudiantes de otros países, así como con otras instituciones de Educación Superior.

Para los ministros «la construcción del Área de Educación Superior Europea es una condición para mejorar el atractivo y la competitividad de las instituciones de Educación Superior en Europa» (Comunicado de Praga, 2001). Resulta determinante la creación de dos grupos de trabajo: el grupo de seguimiento de Bolonia y el grupo preparatorio.

También la European Association for Quality Assurance in Higher Education (en adelante ENQA) comienza a colaborar en el Proceso de Bolonia:

«Los Ministros apelaron a las universidades y a otras instituciones de educación superior, a agencias estatales y a la Red Europea de Garantía de Calidad en la Educación Superior (ENQA), en cooperación con los cuerpos correspondientes de otros países los cuales no son miembros de ENQA, a colaborar en el establecimiento de un marco de trabajo común de referencia y a difundir la mejor práctica» (Declaración de Praga, 2001).

El proyecto del EEES sigue avanzando, los ministros de educación se reúnen en el año 2003 en Berlín, en dicho encuentro se habla de la educación superior como un bien de responsabilidad pública, cuyo objetivo es preservar la riqueza cultural europea. La garantía de calidad en educación superior yace con cada institución en sí misma. El EEES busca la transparencia y la calidad educativa a nivel nacional e institucional. Y se propone seguir trabajando en las siguientes líneas:

- Establecimiento de criterios compartidos en educación superior, así como metodologías compartidas.

- Se determina que para el año 2005 los sistemas de garantía de calidad nacionales deberán incluir:

- Una definición de las responsabilidades de los cuerpos e instituciones involucradas.

- Modelos de evaluación de programas e instituciones.

- Sistemas de acreditación, certificación o procedimientos similares que aseguren la calidad.

- Participación y cooperación internacional y un incremento en la movilidad especialmente en jóvenes investigadores.

- La implantación de dos ciclos en los estudios universitarios: grado y postgrado.

- Elaborar un marco europeo de cualificaciones compatibles y comparables

- Currículum internacional por medio del sistema de ECTS.

- Desarrollo de un sistema de becas para estudiantes con pocas o nulas posibilidades económicas, y un acercamiento al estudiante y sus necesidades.

Vemos que en este encuentro de 2003 hay un cambio con respecto a los anteriores, ya no nos encontramos únicamente con declaraciones de intenciones, sino con objetivos 
concretos que deben alcanzarse, esto implica a los países miembros y sus instituciones que deben ponerse a trabajar en los cambios establecidos. No son cambios menores los que implican al Espacio Europeo de Educación Superior, sino que son cambios que generarán un nuevo sistema universitario para todos aquellos Estados miembros que formen parte del EEES. También la ENQA adquiere un papel relevante en el proceso de Bolonia, vemos que cada vez es más evidente la preocupación por el aseguramiento de la calidad y junto a ella la presunción de que ENQA está dispuesta y capacitada para asumir el papel de organización líder en el aseguramiento de la calidad educativa europea (ENQA, 2010).

Un nuevo encuentro de ministros de educación en 2005 en Bergen da continuidad al proyecto del EEES. En el año 2005 se observan progresos sustanciales en los aspectos prioritarios definidos en el anterior encuentro: la estructura en ciclos ya se materializa en estudios concretos y los sistemas de garantía de calidad comienzan a tomar forma creando trabajo en red entre países, se ha avanzado en el reconocimiento de títulos y periodos de estudios, así como en la elaboración de los marcos de cualificaciones nacionales compatibles con el marco general de cualificaciones del EEES cuyos descriptores generales estarían basados en resultados del aprendizaje y competencias para cada ciclo. No obstante, también se detectan carencias, fundamentalmente en la participación de estudiantes y en la cooperación internacional. Como desafíos y prioridades a trabajar se determinan las siguientes cuestiones:

- Reforzar la investigación y la innovación, esto implicará que las cualificaciones del nivel doctoral se correspondan con el marco global de las cualificaciones del EEES.

- Poner en marcha marcos de cualificaciones nacionales que sean compatibles con el marco global de cualificaciones del EEES.

- Aplicar mecanismos sistemáticos (estándares y directrices) de garantía de calidad.

- Mejorar la accesibilidad a la educación superior, evitando obstáculos socioeconómicos para el estudio.

- Mejorar la cooperación internacional y ampliar la expedición y reconocimiento de títulos conjuntos, incluido el doctorado.

- Mejorar la oferta de becas y créditos para el incremento de la movilidad de estudiantes e investigadores, así como la oferta de itinerarios flexibles de aprendizaje.

En este mismo encuentro de 2005, se determina que el Espacio Europeo de Educación Superior debe estar establecido para el año 2010 comprometiéndose, los implicados en el Proceso, a coordinar las políticas de los Estados para alentar la consecución de los objetivos del proceso. También para el año 2010 se debe trabajar para favorecer la calidad y la transparencia, así como alcanzar la autonomía necesaria para poner en marcha las reformas acordadas.

En el año 2007 se celebra un nuevo encuentro Ministros de Educación en torno al EEES, esta vez en Londres. El trabajo de este encuentro sigue avanzando en la misma línea que los anteriores encuentros, ya se han producido grandes avances; la misión es entonces garantizar que desde las instituciones de educación superior se asuma: 
«La preparación de los estudiantes como ciudadanos activos en una sociedad democrática; la preparación de los estudiantes para su futuro profesional y capacitarles para su desarrollo personal; la creación y conservación de una extensa base de conocimiento avanzado; y el fomento de la investigación y la innovación» (Londres, 2009).

En este encuentro se abordan temas tales como la autonomía institucional, la libertad académica, la importancia de la igualdad de oportunidades, la necesidad de velar por el mantenimiento de instituciones de educación superior fuertes, autónomas, responsables y adecuadamente financiadas. Los ministros de educación consideran que las agencias de garantía de calidad serán determinantes para mejorar la confianza en la educación superior. Las líneas de acción previstas son:

- Continuar promoviendo la movilidad de estudiantes e investigadores. Se considera que existen muchas dificultades burocráticas para la movilidad, en esta línea, se propone superar los obstáculos existentes estableciendo un sistema de becas y prestamos.

- Mejorar la empleabilidad de los egresados

- Intensificar la recogida de datos tanto de la movilidad como de la empleabilidad de los egresados.

- Promover itinerarios flexibles de aprendizaje y seguir trabajando en el reconocimiento de cualificaciones generando una red de trabajo y buenas prácticas que lo apoyen. En este sentido se comprometen a poner en marcha los marcos nacionales de cualificaciones para el año 2010, los cuales tendrán como marco el modelo global del Marco de Cualificaciones del EEES.

- Trabajar desde las Instituciones de educación superior desde un punto de vista del aprendizaje a lo largo de la vida.

- Continuar las líneas de trabajo en materia de calidad educativa. Ya se cuenta con un Registro Europeo de Agencias Certificadoras de Calidad de la Enseñanza Superior lo cual es un gran avance.

- Mejorar la información pública disponible del EEES para dar a conocer de manera global la estrategia llevada a cabo.

Los ministros de educación superior se reúnen nuevamente en el año 2009 en Leuven, Bélgica. Siendo este el último encuentro de la década, los Estados revisan los logros alcanzados y los retos pendientes a los que se deberán hacer frente ya en la nueva década y que se resumen en los diez siguientes elementos clave:

- Dimensión social: se deberá trabajar en la eliminación de las barreras existentes para el acceso a la educación superior. Cada país deberá establecer los mecanismos oportunos para asegurar la equidad en el acceso.

- Aprendizaje a lo largo de la vida: se fomentará en todas las instituciones este nuevo paradigma educativo que implica, en cualquier caso, facilitar al estudiante vías flexibles de aprendizaje en las que tendrán un peso determinante el Marco Europeo de Cualificaciones para el aprendizaje permanente. 
- Empleabilidad: la enseñanza superior deberá preparar adecuadamente a los egresados para la vida laboral. Tiene que trabajarse, por tanto, en las relaciones entre las universidades, empleadores y el gobierno, a fin de que las instituciones de educación superior puedan hacer frente a las necesidades formativas de sus estudiantes.

- Enseñanza centrada en el estudiante: el hecho de poner en el centro del proceso de aprendizaje al propio estudiante requiere de reformas curriculares y de nuevos enfoques de enseñanza. En este punto debe prestarse atención al aseguramiento de la calidad educativa.

- Investigación e innovación: sería deseable que el número de personas con competencias investigadoras aumentara para la próxima década. Conviene, por tanto, trabajar en el reclamo de la investigación y la innovación.

- Cooperación internacional: bajo la creencia de que «el atractivo y la apertura de la educación superior europea se destacará mediante acciones europeas conjuntas» una acción clave será seguir trabajando en la cooperación educativa intergubernamental.

- Movilidad: la movilidad es el sello distintivo del Espacio Europeo de Educación Superior, por este motivo, y por todos los elementos positivos asociados a la movilidad, se insta a los países participantes a aumentar la movilidad (tanto de docentes como de estudiantes) y asegurar una alta calidad de la misma. Para 2020 al menos un 20 \% de los graduados europeos deberán haber tenido un periodo de estudio en el extranjero.

- Recogida de datos: la recogida de datos permitirá conocer la evolución y realizar la supervisión de los progresos realizados.

- Mecanismos de transparencia: la transparencia del EEES está vinculada a los sistemas de garantía de calidad, estos son una prioridad en los países e instituciones implicados.

- Financiamiento de la educación superior: la financiación pública garantizará el acceso a los sistemas de educación superior, se anima a los Estados a que busquen nuevas fuentes de financiación.

Desde la Declaración de la Sorbona hasta la Conferencia de ministros de educación superior de 2009 en Leuven se han descrito siete encuentros, como decíamos al comienzo todos ellos determinantes para la puesta en marcha del EEES. En esta primera década se han dado los primeros pasos, se han sentado las bases, se han creado grupos de trabajo, se han realizado informes y se ha instado a los países participantes a que pongan en marcha en sus instituciones el EEES.

El año 2010 era el plazo que los ministros habían dado a los países para hacer los cambios establecidos. A continuación, abordaremos las cuestiones más relevantes del EEES para la segunda década del milenio.

\subsection{El afianzamiento del Espacio Europeo de Educación Superior (2010-2020)}

El primer encuentro de ministros de esta década se produce el mismo año 2010 en Budapest. En él se subrayan los logros alcanzados hasta el momento, tales como la cooperación europea entre organismos e instituciones; la comparabilidad entre los sistemas 
de educación superior que favorecen la movilidad; la creación de las agencias de calidad europeas y el aumento de la transferencia, transparencia y reconocimiento de créditos. Pero también se hace eco, por vez primera, de las quejas surgidas en algunos países derivadas de la puesta en marcha del mismo proceso, a las que se debe atender y dar respuesta: «reconocemos y escucharemos las voces críticas surgidas entre el personal y los estudiantes» (Budapest, 2010).

La década comienza con dificultades en la implantación de los cambios, pero de esta primera reunión ministerial podemos rescatar el llamamiento, que se lanza a los países involucrados, a seguir trabajando por el buen funcionamiento del EEES. El impulso del proceso de Bolonia sigue adelante, y se solicita al Grupo de Seguimiento de Bolonia que proponga medidas que contribuyan a la correcta implantación tanto de los principios como de las líneas de acción acordadas para el EEES en los sistemas nacionales de educación superior y en sus instituciones. Algunas de las propuestas son: desarrollar métodos de trabajo complementarios que apoyen la puesta en marcha del proceso, realizar visitas de estudio u otro tipo de actividades que fomenten el intercambio de información.

En el año 2012, los ministros de Educación Superior se encuentran en Bucarest en el marco del EEES, la crisis económica que atraviesa Europa en estos momentos comienza a tener repercusiones también en la educación superior «la crisis está afectando la disponibilidad de fondos adecuados y haciendo que las perspectivas laborales de los graduados sean más inciertas» (2012, p.1). Sin embargo, existe también la convicción de que la solución a estos problemas económicos está (en parte) vinculada a la educación. Siendo este el contexto, en el comunicado de Bucarest se recoge la preocupación por asegurar el mayor financiamiento público para la educación superior, por mejorar la empleabilidad de los egresados, reducir el desempleo juvenil y por consolidar los avances logrados hasta el momento: los sistemas de garantía de calidad, el sistema de créditos, las convalidaciones, el suplemento europeo al título, la estructura de tres ciclos, el aprendizaje centrado en el estudiante y los sistemas de movilidad.

Para el periodo 2012-2015 pondrán especial atención en establecer la implementación integral del Plan Bolonia en todas las instituciones, centrándose en:

- Asegurar la finalización de los estudiantes, así como la incorporación de métodos de enseñanza innovadores que tengan los resultados de aprendizaje como meta del proceso educativo y que estos, a su vez, estén vinculados con los créditos de estudio. De la mano de esta cuestión encontramos la mejora de los marcos de cualificaciones y la empleabilidad de los egresados

- Seguir trabajando en los sistemas de garantía de calidad nacionales a través de las agencias creadas para tal fin.

- Asegurar un vínculo más estrecho con la investigación, ofreciendo una variedad de programas de doctorado.

Tres años después, los ministros reunidos en Yereván (Armenia) en el año 2015 se congratulan de reconocer que el EEES es ya una realidad en cuarenta y siete países con diferentes tradiciones (políticas, culturales y académicas) que han cooperado sobre la base del diálogo y han alcanzado objetivos compartidos por medio de compromisos comunes: 
«Para el año 2020 estamos decididos a lograr un EEES en el que nuestros objetivos comunes se apliquen en todos los países miembros para garantizar la confianza mutua en los sistemas de educación superior, donde el reconocimiento automático de las calificaciones se ha convertido en una realidad para que los estudiantes y graduados puedan moverse fácilmente a través de él; donde la educación superior está contribuyendo eficazmente a la construcción de sociedades inclusivas, fundadas en los valores democráticos y los derechos humanos; y donde las oportunidades educativas proporcionan las competencias y aptitudes necesarias para la ciudadanía europea, la innovación y el empleo» (Consejo de Ministros de Educación Superior, 2015).

En este encuentro en Yereván son cuatro las metas que se plantean alcanzar:

- Seguir mejorando la calidad y la relevancia del aprendizaje y de la enseñanza.

- Fomentar la empleabilidad de los egresados a lo largo de toda su vida laboral, no solo al terminar los estudios.

- Hacer que los sistemas de educación superior sean cada vez más inclusivos dada la diversidad creciente de los países.

- La implementación completa y coherente de las reformas acordadas a nivel nacional, así como el compromiso de los agentes políticos implicados en ello.

El encuentro de ministros más reciente tuvo lugar en 2018 en París, en este último encuentro se revisa nuevamente el progreso del proceso de Bolonia en los países, observando una implementación desigual tanto entre áreas de política como entre países. Se insiste, en consecuencia, en la importancia del aseguramiento de la calidad, el documento «Estándares y directrices para el aseguramiento de la calidad en el EEES», se recuerda, está a disposición de los países para orientar las reformas. En esta línea de la calidad se da la bienvenida a DEQUAR ${ }^{1}$, una base de datos de informes externos de garantía de calidad para todos los países del EEES, que facilitará la comparabilidad, reconocimiento, movilidad, intercambio y transparencia.

Coinciden en centrarse, para el periodo de tiempo 2018-2020, en tres compromisos clave: (1) completar la implantación del sistema de tres ciclos compatible con los ECTS y las cualificaciones del EEES,(2) el cumplimiento del Convenio sobre Reconocimiento de Cualificaciones relativas a la Educación Superior en la Región Europea ${ }^{2}$ y (3) cumplir lo indicado en el documento Normas y Directrices para la Garantía de la Calidad en el Espacio Europeo de Educación Superior (ESG, 2015). También, para este periodo de tiempo se seguirá trabajando en la cooperación y la movilidad, en mejorar la calidad de la enseñanza y el aprendizaje incidiendo (como ya se hizo anteriormente) en el aprendizaje permanente y en la innovación educativa. Se mejorarán las sinergias en el triángulo investigación - educación - innovación. Se trabajará en hacer un mejor uso de la educación digital (cuestión en la que se desea hacer una mayor profundización). Otra cuestión de enorme importancia, y de la que se ha hablado poco a lo largo del proceso de Bolonia es la formación del profesorado, en esta reunión los ministros la abordan en

1 DEQAR es la base de datos de resultados externos de garantía de calidad, cuyo objetivo es apoyar la transparencia de la calidad de la educación superior europea y su gestión. DEQAR fue seleccionado para la cofinanciación de la UE por el programa Erasmus + de la Unión Europea.

2 Es el principal texto jurídico internacional que tiene por objeto el justo reconocimiento de las cualificaciones en educación superior. Fue elaborado en 1997 por el Consejo de Europa y la UNESCO. 
los siguientes términos: "promoveremos y apoyaremos iniciativas institucionales, nacionales y europeas para la formación pedagógica y desarrollo profesional continuo de los docentes de educación superior y exploraremos formas para un mejor reconocimiento de la innovación y de la calidad en su carrera» (París, 2018, p. 4).

Los representantes ministeriales de los países participantes en el EEES quedaron emplazados para un nuevo encuentro en Italia para el año 2020, dicho encuentro de ministros no ha llegado a producirse todavía debido a la situación de pandemia mundial producida por el COVID-19. Sin embargo, a pesar de la suspensión del encuentro, se sigue trabajando en el EEES, ya en París (2018) se «miró» más allá de 2020 y se planteó un EEES más ambicioso, predispuesto a una mayor cooperación interdisciplinaria y transfronteriza, con la mano tendida para crear lazos con el Área Europea de Investigación y el Comité de Innovación (ERAC), con el Espacio Europeo de Investigación (EEI), con los Objetivos de Desarrollo Sostenible (ODS) de las Naciones Unidas. «Reconocemos que se requiere un mayor esfuerzo para fortalecer la dimensión social de la educación superior» (2018, p. 4).

En el proceso de Bolonia, como hemos visto, el modelo del EEES es hoy un modelo de cooperación intergubernamental estructurado, un modelo de diálogo y de trabajo en red. Se apostó por la mejora de la educación y, en la actualidad, vemos que el proceso de Bolonia ha dado frutos.

\subsection{La calidad como elemento clave en el EEES}

Resulta interesante, una vez realizada la revisión histórica del proceso, hacer un pequeño análisis de los documentos con que se ha trabajado, así a modo de análisis cuantitativo hemos querido hacer un conteo de las palabras más repetidas en las comunicaciones y declaraciones emitidas en los diferentes encuentros de ministros de educación superior. Se ha utilizado para ello el programa de análisis Atlas.ti que, mediante el examinador de palabras, nos ha permitido reconocer las palabras más repetidas a lo largo de estos 20 años de reuniones ministeriales. El programa permite eliminar elementos lingüísticos sin valor referencial tales como preposiciones, artículos, etc., también permite filtrar palabras que no se desean considerar en el análisis.

Reconocer las palabras más repetidas, como podemos observar en la figura 1, puede aportar valor, dado que, la importancia de las palabras es innegable en cualquier disciplina vinculada a las ciencias sociales, y cabe deducir que el número de veces que aparezcan ciertas palabras es proporcional a su importancia dentro del proceso. (Hernández, 2015). En este mismo sentido Colella y Díaz-Salazar (2014) afirman que es a partir de los sintagmas desde donde le damos sentido al discurso. A la luz de la Figura 1 podemos ver que determinadas palabras se repiten con frecuencia en los comunicados ministeriales del Proceso de Bolonia, mostrando así la importancia de determinados aspectos. Resulta previsible encontrar repetidas palabras tales como: superior, Europa, Bolonia, educación, área, EEES o instituciones, que por razones evidentes han aparecido en los textos más de 150 veces en casi todos los casos. Otros términos que han aparecido frecuentemente y que merece la pena destacar han sido (por orden): estudiante/s (154), aprendizaje (150), calidad (138), desarrollo (126), reconocimiento (119) y cualificación/ es (106), tal y como se muestraen la Figura 1. 


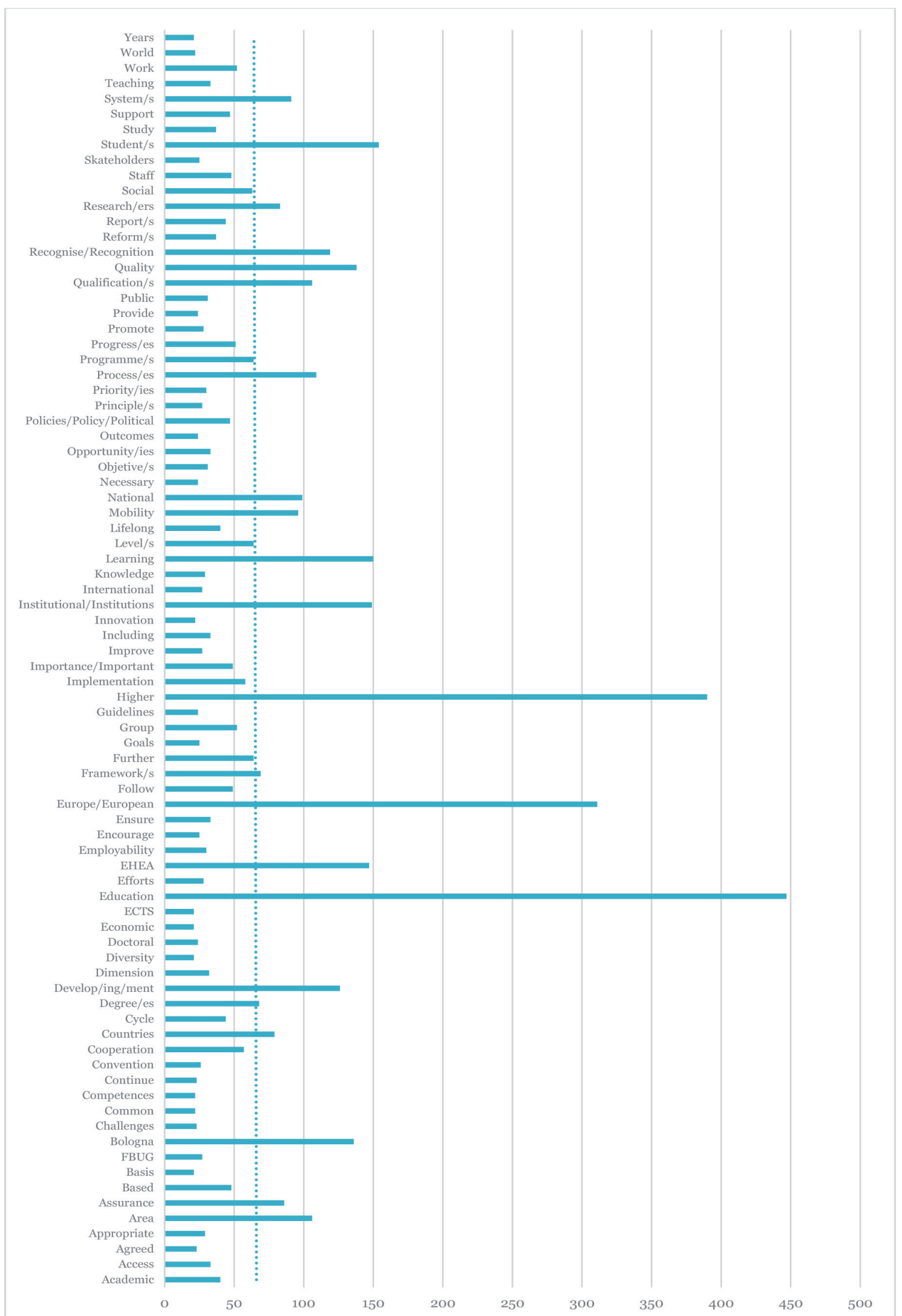

Figura 1. Palabras más repetidas en los documentos del Proceso de Bolonia. Fuente: Elaboración propia. 
«Resulta también lógico encontrar entre estas los términos «estudiante/s» pues son ellas y ellos quienes vivirán las transformaciones derivadas del Proceso, o «aprendizaje» dado que, claramente, la tendencia impactará en los procesos de aprendizaje de los estudiantes incorporando ahora los denominados «resultados de aprendizaje» que serán un componente esencial en los procesos de enseñanza-aprendizaje, contribuyendo todo ello a un cambio de paradigma a favor de la enseñanza y el aprendizaje centrados en el estudiante» (ESG, 2015).

El término «calidad», por su parte, se suma a esta lista de palabras más repetidas en las declaraciones y comunicaciones ministeriales. Lo cual es reflejo de la preocupación por las cuestiones de calidad en las instituciones de educación superior de los países de EEES. En todos los encuentros ministeriales aparece tanto de manera directa como de manera indirecta la cuestión de la calidad dado que «la aspiración de mejorar la calidad de la oferta de la educación terciaria en todo el Espacio Europeo de Educación Superior es un elemento clave dentro del Proceso de Bolonia» (Comisión Europea, 2015, p. 89). La calidad educativa es una cuestión que ha suscitado un gran interés en el ámbito académico por ser un tema de enorme complejidad, de gran calado y que genera controversias sobre su sentido, su medición y su implantación. Numerosos autores han tratado de dilucidar su concepto y su alcance, concluyendo que se trata casi de una cuestión política-filosófica en la que se pueden identificar diversas concepciones (Egido y Haug, 2006; Cascante, 2009; MuñozRepiso y Murillo, 2010; López Aguado, 2018; Álvarez-López y Matarranz , 2020).

En el documento denominado Criterios y directrices para el aseguramiento de Calidad en el Espacio Europeo de Educación Superior (ESG) elaborado en 2015; presentada conjuntamente por los siete grupos de interés que conforman el Espacio Europeo de Educación Superior (EEES): EUA, EURASHE, ESU, ENQA, Business Europe, Education International y EQAR; y aprobado por la conferencia de ministros se plantea la calidad en los siguientes términos:

«La educación superior tiene múltiples finalidades, que incluyen preparar a los estudiantes para una ciudadanía activa, para sus futuras profesiones (por ejemplo, contribuyendo a su empleabilidad), ayudar en su desarrollo personal, crear una amplia base de conocimientos avanzados y fomentar la investigación y la innovación. Por lo tanto, los grupos de interés, que pueden dar prioridad a los diferentes objetivos, pueden ver la calidad de la educación superior de manera diferente $\mathrm{y}$, por consiguiente, el aseguramiento de la calidad debe tener en cuenta los distintos puntos de vista. La calidad, aunque no es fácil de definir, es principalmente el resultado de la interacción entre profesores, estudiantes y el entorno de aprendizaje institucional. El aseguramiento de la calidad debe garantizar un entorno de aprendizaje en el que el contenido de los programas, las oportunidades de aprendizaje y los recursos se ajusten a sus fines». (2015, pp.8-9)

Como vemos, la calidad en el EEES se plantea como un elemento de responsabilidad y de mejora para la educación superior, es decir, para dar confianza a docentes, estudiantes y personal sobre la formación que ofrecen las instituciones y para realizar seguimientos y acciones de mejora que optimicen los procesos formativos. 


\section{La calidad como el sello inequívoco del EEES}

Una vez realizado el recorrido por los hitos más relevantes del proceso de Bolonia, podemos dar un paso más y reconocer que uno de los elementos más significativos en el EEES es la puesta en marcha de mecanismos para garantizar la calidad en la educación superior de los países participantes. Tal y como veíamos en el anterior epígrafe, la calidad ha sido uno de los elementos más repetidos en las reuniones ministeriales, muestra de la importancia tan decisiva que tiene en el EEES. Han pasado veinte años desde el primer encuentro que originó el proceso de Bolonia y, desde entonces, el discurso de la calidad educativa ha tomado mucha fuerza, en esta línea Fernández-González y Monarca (2018) subrayan la centralidad de la calidad en los discursos de política educativa; también Colella y Díaz-Salazar (2014) coinciden en que «el discurso de la calidad domina el exceso de sentido que supone la discursividad de lo educativo, convirtiéndose esa fijación parcial del sentido en el modo legítimo -y normativo- del ser-educación en la actualidad» (p.299). La calidad educativa se ha convertido en el centro de atención de las políticas educativas, no solo en el contexto del EEES sino como tendencia a nivel global, de hecho, el objetivo 4 de la Agenda 2030 para el Desarrollo Sostenible consiste en «garantizar una educación inclusiva y equitativa de calidad y promover oportunidad de aprendizaje permanente para todos» (UNESCO, 2015, p. 5)

Los mecanismos de calidad que se han puesto en marcha en el EEES han sido varios y de gran impacto en las instituciones universitarias, en esta misma línea la agencia de calidad ANECA (2018) afirma que:

«las políticas, los instrumentos y los procesos que, en relación a la garantía de la calidad universitaria, ponen en funcionamiento los gobiernos, las universidades y las agencias de evaluación, están al servicio de los objetivos dados a la educación universitaria en marcos generales como los propuestos desde foros como el Espacio Europeo de Educación Superior -EEES-» (p. 7)

El siguiente mapa de la Figura 2 ilustra la implementación, por parte de los países, del compromiso clave en el aseguramiento externo de la calidad en todo el Espacio Europeo de Educación Superior (EEES). Todas las instituciones de educación superior están sujetas a una garantía externa de calidad que se regula por medio de agencias que han demostrado con éxito el cumplimiento de las Normas y Directrices para la Garantía de Calidad $^{3}$ en el EEES mediante el registro en EQAR ${ }^{4}$

3 Normas y Directrices para la Garantía de la Calidad en el Espacio Europeo de Educación Superior (European Standards and Guidelines for Quality Assurance in the European Higher Education Area; ESG por sus siglas en inglés)

4 El Registro Europeo de Garantía de Calidad para la Educación Superior (EQAR) es el registro oficial de agencias de garantía de calidad del Espacio Europeo de Educación Superior que cumplen sustancialmente con el ESG. Puede accederse a la base de datos a través de su página web: https://www.eqar.eu/.Todas las agencias oficiales de los países pertenecientes al EEES, por tanto, deben estar registradas en EQAR. 


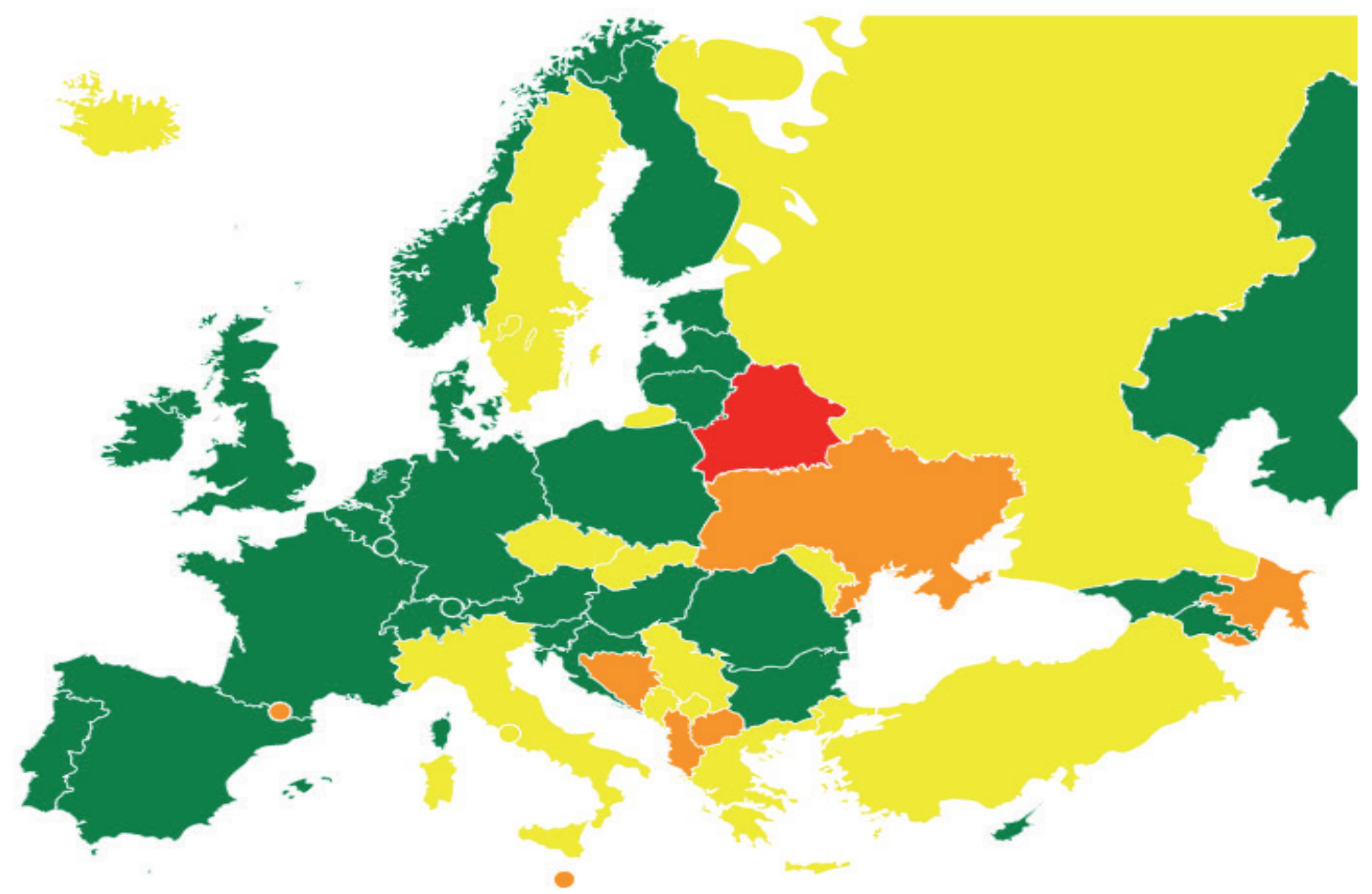

Tiene un sistema de garantía de calidad funcional en todo el país, las instituciones de educación superior están sujetas a una garantía externa de calidad por parte de agencias registradas en EQAR.

Tiene un sistema de garantía de calidad funcional en todo el país, pero solo algunas instituciones de educación superior están sujetas a una garantía externa de calidad por parte de agencias registradas en EQAR. ESG.

No tiene implantado ningún sistema de garantía de calidad funcional.

Figura 2. Países del Espacio Europeo de Educación Superior Fuente: EQAR (2020)

Cada uno de los países participantes en el EEES ha puesto en funcionamiento sistemas de garantía de calidad, siguiendo así la pauta por procurar la mejora de la calidad entre las prioridades que se destacan en los comunicados de ministros de educación superior. Pero, dentro de estas acciones de aseguramiento de calidad hay todavía cierta capacidad de decisión por parte de las instituciones de educación superior del EEES. Tal y como podemos ver en la Tabla 1 cada uno de los países del EEES tiene sus propias preferencias con respecto al aseguramiento de la calidad. 
Tabla 1.

Acreditaciones y agencias oficiales en los países del EEES

\begin{tabular}{|c|c|c|c|c|c|c|c|}
\hline \multirow{2}{*}{ PAís } & \multirow{2}{*}{$\begin{array}{c}\text { ALINEAMIENTO } \\
\text { CON EL ESG } \\
\end{array}$} & \multirow{2}{*}{$\begin{array}{l}\text { AGENCIAS } \\
\text { OFICIALES } \\
\end{array}$} & \multirow{2}{*}{$\begin{array}{l}\text { INSTITUCIONES } \\
\text { CON INFORMES } \\
\end{array}$} & \multicolumn{2}{|c|}{ TIPO } & \multicolumn{2}{|c|}{ ALCANCE } \\
\hline & & & & OBLIGATORIA & VOLUNTARIA & INSTITUCIONES & $\begin{array}{c}\text { PROGRAMAS } \\
5\end{array}$ \\
\hline Armenia & $\square$ & 7 & 13 & 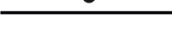 & 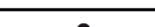 & 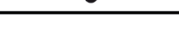 & 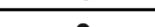 \\
\hline \multirow{2}{*}{ Austria } & \multirow{2}{*}{$\square$} & \multirow{2}{*}{14} & \multirow{2}{*}{24} & $\bullet$ & & $\bullet^{6}$ & $\bullet 7$ \\
\hline & & & & & $\bullet$ & & $\bullet$ \\
\hline Bélgica & 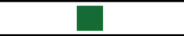 & 9 & 38 & $\bullet$ & & $\bullet^{8}$ & $\bullet$ \\
\hline Bulgaria & $\square$ & 5 & 2 & $\bullet$ & & $\bullet$ & $\bullet$ \\
\hline Croacia & $\square$ & 4 & 119 & $\bullet$ & & $\bullet$ & $\bullet$ \\
\hline Chipre & $\square$ & 6 & 4 & $\bullet$ & & $\bullet$ & $\bullet$ \\
\hline Dinamarca & $\square$ & 5 & 28 & $\bullet$ & & $\bullet$ & $\bullet$ \\
\hline Estonia & $\square$ & 5 & 21 & $\bullet$ & & $\bullet$ & - \\
\hline Finlandia & $\square$ & 4 & 43 & $\bullet$ & & $\bullet 9$ & \\
\hline Francia & $\square$ & 11 & 274 & - & & $\bullet$ & \\
\hline Georgia & $\square$ & 3 & 38 & $\bullet$ & & - & $\cdot \bullet^{10}$ \\
\hline \multirow{2}{*}{ Alemania } & \multirow{2}{*}{$\square$} & \multirow{2}{*}{17} & 66 & - & & & - \\
\hline & & & 309 & & $\bullet$ & - & \\
\hline Hungría & L & 7 & 4 & $\bullet$ & & - & $\bullet$ \\
\hline Irlanda & $\square$ & 9 & 2 & & $\Delta^{11}$ & $\diamond$ & $\diamond$ \\
\hline Kazajstán & 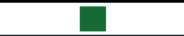 & 12 & 65 & - & & - & $\bullet$ \\
\hline Letonia & $\square$ & 3 & 1 & - & & $\bullet$ & \\
\hline Liechtenstein & $\square$ & 4 & 1 & - & & $\bullet$ & - \\
\hline Lituania & $\square$ & 7 & 2 & - & & $\bullet$ & $\bullet$ \\
\hline Luxemburgo & $\square$ & 7 & 1 & $0^{12}$ & & - & - \\
\hline Países Raios & $\pi$ & 11 & 6 & - & & & - \\
\hline Palses bajos & $\mathbf{D}$ & 11 & 0 & & - & - & \\
\hline Noruega & $\square$ & 5 & 3 & $\bullet$ & & $\diamond^{13}$ & $\bullet^{14}$ \\
\hline Polonia & $\square$ & 7 & 316 & $\bullet$ & & & $\bullet$ \\
\hline Portugal & $\square$ & 8 & 94 & $\bullet$ & & $\bullet$ & $\bullet$ \\
\hline Rumania & $\square$ & 8 & 91 & - & & - & $\bullet$ \\
\hline Eslovenia & $\square$ & 12 & 51 & - & & - & - \\
\hline Fsnaก̃a & 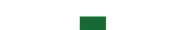 & 15 & $6-9$ & - & & & - \\
\hline Lspanta & 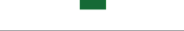 & 15 & 054 & & - & - & \\
\hline Suiza & 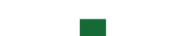 & 12 & 12 & - & & - & $\bullet^{15}$ \\
\hline Nalza & & 12 & 13 & & - & & - \\
\hline Reino Unido & $\square$ & 6 & 14 & - & & 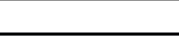 & \\
\hline $\begin{array}{c}\text { República } \\
\text { Checa }\end{array}$ & $\square$ & 9 & 5 & $\bullet$ & & $\bullet$ & \\
\hline Grecia & & 3 & 3 & $\diamond^{16}$ & & & \\
\hline Santa Sede & & - & - & & & & \\
\hline Islandia & & 1 & 11 & & & & \\
\hline Italia & & 6 & 2 & - & & - & - \\
\hline Kosovo & & 4 & 3 & & & & \\
\hline Moldavia & & 4 & 14 & - & & - & $\bullet$ \\
\hline Montenegro & & 1 & 9 & - & & - & - \\
\hline Rusia & 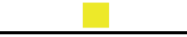 & 14 & 38 & - & & - & - \\
\hline Serbia & & 4 & 1 & & & & \\
\hline Eslovaquia & & 2 & - & - & & - & \\
\hline Suecia & & 6 & 3 & & & & \\
\hline Turquía & & 6 & 8 & - & & - & \\
\hline Albania & $\square$ & 3 & 4 & - & & $\bullet$ & $\bullet$ \\
\hline Andorra & 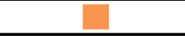 & 1 & - & & & & \\
\hline Azerbaiyán & $\square$ & 2 & 3 & - & & - & - \\
\hline $\begin{array}{c}\text { Bosnia y } \\
\text { Herzegovina }\end{array}$ & 口 & 5 & 3 & $\bullet$ & & $\bullet$ & $\bullet$ \\
\hline Malta & 口 & 2 & 1 & $\bullet$ & & $\bullet$ & $\bullet$ \\
\hline $\begin{array}{c}\text { Macedonia } \\
\text { del Norte }\end{array}$ & 口 & 2 & 6 & & & & \\
\hline Ucrania & $\square$ & 5 & 2 & $\bullet$ & & & $\bullet$ \\
\hline Bielorrusia & & - & - & $\diamond^{17}$ & & & \\
\hline
\end{tabular}

Fuente: Elaboración propia a partir de los datos de EQAR (2020) 
Tal y como se puede apreciar en la Tabla 1, los mecanismos que más relevancia han tomado en el EEES son, por un lado, la creación de agencias de calidad nacionales e incluso regionales, además de la ya existente ENQA desde el comienzo del proceso de Bolonia, la cual alberga y orienta las acciones de todas las agencias de calidad que posteriormente han ido surgiendo. Por otro lado, la aparición de numerosos procedimientos de evaluación externa por parte de dichas agencias a instituciones de educación superior y a programas educativos de dichas instituciones.

La Tabla 1 refleja el panorama general de los países del EEES y sus respectivas agencias de calidad que realizan evaluaciones externas; en la tabla vemos que la gran mayoría de los países (incluso los que todavía no tienen sus sistemas de calidad alineados completamente ni parcialmente con el ESG) llevan a cabo, de manera obligatoria, evaluaciones externas bien de instituciones, bien de programas educativos, siendo en muchos de los casos, imprescindible la obtención de un informe de evaluación favorable para el funcionamiento de las instituciones o el desarrollo e implantación de los programas educativos. Y que en muy pocos casos las evaluaciones externas son opcionales y elegidas de manera voluntaria por las instituciones. En concreto, son treinta y tres los países que tienen implantados sistemas de evaluación externa para las instituciones educativas de manera obligatoria y treinta y uno los que tienen la evaluación obligatoria para los programas educativos; por su parte y en contra de la tendencia, son solo tres países los que proponen estas evaluaciones externas como opcionales tanto para instituciones como para programas.

5 Solo es obligatoria en programas académicos médicos.

6 Solo es obligatoria en instituciones públicas y en ciencias aplicadas.

7 Solo es obligatoria en programas de ciencias aplicadas y en programas de instituciones privadas.

$8 \mathrm{La}$ acreditación institucional solo es obligatoria para la comunidad flamenca belga.

9 En Finlandia es considerado como un proceso obligatorio de auditoría.

10 Obligatoria para programas de doctorado y programas que den acceso a profesiones reguladas.

11 En Irlanda los procesos de acreditación son complementarios a los procedimientos de calidad ya existentes en las instituciones.

12 Aunque en Luxemburgo los procesos de acreditación son obligatorios para instituciones y programas, la última palabra sobre los resultados de los informes de acreditación la tiene el ministerio de educación.

13 En Noruega las instituciones tienen la posibilidad de autoacreditarse.

14 Es obligatoria la acreditación del programa cuando la institución a la que pertenece dicho programa no esté acreditada.

15 La acreditación es obligatoria para los programas relativos a profesiones sanitarias, para el resto de los programas académicos es voluntaria.

16 Aunque la acreditación es obligatoria, se puede elegir entre la acreditación de la institución o de los programas.

17 En Bielorrusia la acreditación la da el Estado, no las agencias de calidad. 


\section{Conclusiones}

A continuación, se proponen algunas reflexiones a modo de cierre derivado del recorrido realizado a lo largo del presente trabajo. Comenzábamos revisando la historia del EEES con sus respectivos encuentros ministeriales y los pequeños avances que han permitido la configuración del mismo. Nos hemos adentrado posteriormente en la calidad de la educación desde la perspectiva que se propone en el EEES.

En primer lugar, cabe reconocer que el proceso de Bolonia, y en concreto el EEES es un proyecto único y ejemplo de cooperación intergubernamental. El Proceso de Bolonia ha sido, visto en perspectiva, un éxito y ha demostrado que se puede trabajar de manera cooperativa desde el respeto y el diálogo. Se han producido grandes cambios en los sistemas de educación de los países miembros y, aunque evidentemente el EEES tiene todavía margen de mejora, los cambios que ha generado en educación superior pueden considerarse claramente positivos. El trabajo mantenido en el tiempo a través de las reuniones ministeriales, los grupos de trabajo que se han ido creando y todos los agentes implicados en los países miembros han permitido la creación de esos mecanismos transformadores de la educación superior haciendo que el sistema universitario haya cambiado de manera determinante en los últimos años y de manera más significativa en la última década.

El EEES es, también, un claro ejemplo de Política Educativa Supranacional, siendo como hemos visto una política educativa que, nacida en el seno de la Unión Europea como institución, se convierte en tendencia mundial y que modifica las políticas educativas (de educación superior en este caso) de las naciones, generando unas políticas educativas comunes y armonizadas. En este caso, además de traspasar las barreras nacionales, ha traspasado a la propia institución de la Unión Europea, dado que hoy son ya cuarenta y ocho países los que forman parte del EEES, participando plenamente del mismo.

El modelo de calidad que se ha propuesto en el EEES ha tenido un innegable impacto político en los países. La perspectiva de la calidad en el EEES es solo una de las múltiples que existen, ya afirmaba Laclau (1996) que la calidad es un concepto que depende absolutamente de la interpretación que se le quiera dar. En nuestro caso, hemos visto que la calidad educativa ha implicado la creación de criterios comunes para los países, así como la implantación de evaluaciones externas. A la luz del estudio realizado, resulta evidente que la rendición de cuentas ha venido para quedarse y, en este sentido, el modelo de calidad educativa que se ha impuesto por medio de los procedimientos establecidos, parece que está dando frutos positivos y que, en mayor o menor medida, está funcionando adecuadamente en todos los países del EEES. La mayor parte de los países han implantado procesos de acreditación obligatoria tanto en sus instituciones de educación superior como en sus programas educativos oficiales que aseguran la calidad de la educación superior, esta armonización nunca vista es buena muestra del papel determinante que ha tenido la calidad en el Proceso de Bolonia. El creciente número de agencias de calidad y el creciente número de informes de acreditación que se han emitido (evaluando a instituciones y programas educativos) es un claro indicador de que la cultura de la calidad ha impactado fuertemente en los países y en las instituciones de educación superior.

Cabe esperar que en un futuro no muy lejano se extienda la obligatoriedad de las evaluaciones externas a otros elementos y/o agentes que forman parte de la cadena de la educación superior. 
Finalmente se propone, como línea de trabajo futuro, la evaluación de los procesos de calidad puestos en marcha hasta el momento, podríamos denominarlo la metaevaluación de los sistemas de garantía de calidad. El fenómeno de la calidad es un fenómeno que ha venido para quedarse, como veíamos anteriormente y, probablemente, necesite todavía recorrer mucho camino para mejorar algunos de sus procedimientos. Junto con los beneficios derivados del sello de calidad del EEES, es importante reconocer también los peligros asociados al excesivo afán del aseguramiento de la calidad, dado que corremos el riesgo de prestar más atención a los procesos de calidad que a la calidad misma, a las evaluaciones externas y sus informes, que a la realidad educativa; a los índices de impacto de las publicaciones, que al contenido de las mismas; a las horas de docencia o a los resultados de las encuestas que al mismo proceso de enseñanza-aprendizaje. No cabe duda, los procesos de aseguramiento de la calidad son una valiosa herramienta que buscan la mejora de la educación superior, pero es necesario que revisemos periódicamente esos modelos de calidad, que recordemos que la educación está en el centro de todos estos procesos y que por tanto los mecanismos y las herramientas de calidad deben estar siempre al servicio de la educación.

\section{Referencias}

Álvarez-López, G.; Matarranz, M. (2020). Calidad y evaluación como tendencias globales en política educativa: estudio comparado de agencias nacionales de evaluación en educación obligatoria en Europa. Revista Complutense de Educación, 31(1), 85-93

ANECA. (2018). Informe sobre el estado de la evaluación externa de la calidad de las universidades españolas. Madrid: ANECA.

Bucharest Communiqué 26th and 27th of april 2012. Making the most of our potential: Consolidating the European Higher Education Area. Disponible en: http://www. ehea.info/page-ministerial-conference-bucharest-2012. Fecha de acceso: 1 de junio de 2020.

Cascante Fernández, C. (2009). ¿Refundar Bolonia? Un análisis político de los discursos sobre el proceso de creación del Espacio Europeo de Educación Superior. Revista Española de Educación Comparada, 15, pp. 131-161.

Colella, L. y Díaz-Salazar, R. (2014). El discurso de la calidad educativa: un análisis crítico. Educ. Educ., 18(2), 287-303. DOI: 10.5294/edu.2015.18.2.6

Comisión Europea/EACEA/Eurydice. (2015). El Espacio Europeo de Educación Superior en 2015: Informe sobre la implantación del Proceso de Bolonia. Luxemburgo: Oficina de Publicaciones de la Unión Europea.

Comunicación de la Comisión al Parlamento Europeo, al Consejo, al Comité Económico y Social Europeo y al Comité de las Regiones, de 20 de septiembre de 2011, Apoyar el crecimiento y el empleo - una agenda para la modernización de los sistemas de educación superior en Europa. COM (2011) 567 final.

Communication from the Commission to the European Parliament, the Council, the European Economic and Social Committee and the Committee of the Regions, 20th september 2011, Supporting growth and Jobs - an agenda for the modernisation of Europe's higher education systems. COM (2011) 1063 final. 
Council recommendation on a Quality Framework for Traineeships. Disponible en: http:// www.consilium.europa.eu/uedocs/cms_data/docs/pressdata/en/lsa/141424.pdf. Fecha de acceso: 6 de noviembre de 2020

Comunicado de la Conferencia de Ministros responsables de la Educación Superior, mantenida en Berlín el 19 de septiembre del 2003. Comunicado de Berlín, 2003. Educación Superior Europea. Disponible en: http://www.ehea.info/pageministerial-conference-berlin-2003. Fecha de acceso: 1 de junio de 2020.

Comunicado de la Conferencia de Ministros Europeos responsables de Educación Superior. Bergen, 19-20 de mayo de 2005. El Espacio Europeo de Educación Superior-Alcanzando las metas. Disponible en: http://www.ehea.info/pageministerial-conference-bergen-2005. Fecha de acceso: 1 de junio de 2020.

Comunicado de Londres, de 18 de mayo de 2007. Hacia el Espacio Europeo de Educación Superior: respondiendo a los retos de un mundo globalizado. Disponible en: http:// www.ehea.info/page-ministerial-conference-london-2007. Fecha de acceso: 1 de junio de 2020.

Comunicado de la Conferencia de Ministros Europeos responsables de educación superior, Leuven / Louvain-la-Neuve, 28 y 29 de abril de 2009. Comunicado de Lovaina, 2009. El proceso de Bolonia 2020- El Espacio Europeo de Educación Superior en la nueva década. Disponible en: http://www.ehea.info/pageministerial-conference-Leuven-Louvain-la-Neuve-2009. Fecha de acceso: 1 de junio de 2020.

Convenio sobre Reconocimiento de Cualificaciones relativas a la Educación Superior en la Región Europea (1997). Disponible en: http://portal.unesco.org/es/ev.php-URL_ ID=13522\&URL_DO=DO_TOPIC\&URL_SECTION=201.html. Fecha de acceso: 12 de junio de 2020.

Declaración conjunta de los ministros europeos de educación reunidos en Bolonia el 19 de junio de 1999. El Espacio Europeo de la Enseñanza Superior. Disponible en: http://www.ehea.info/page-ministerial-conference-bologna-1999. Fecha de acceso: 1 de junio de 2020.

Declaración del encuentro de los Ministros Europeos en funciones de la Educación Superior en Praga, de 19 de mayo de 2001. Declaración de Praga, 2001. Hacia el Área de la Educación Superior Europea. Disponible en: http://www.ehea.info/ page-ministerial-conference-prague-2001. Fecha de acceso: 1 de junio de 2020.

Declaración de Budapest-Viena de 12 de marzo de 2010. Declaración de Budapest, 2010. Sobre el Espacio Europeo de Educación Superior. Disponible en: http://www. ehea.info/page-ministerial-conference-budapest-vienna-2010. Fecha de acceso: 1 de junio de 2020.

Egido Gálvez, I. y Haug, G. (2006). La acreditación como mecanismo de garantía de la calidad: tendencias en el Espacio Europeo de Educación Superior. Revista Española de Educación Comparada, 12, pp. 81-112.

Fernández-González, N. y Monarca, H. (2018). Política educativa y discursos sobre calidad: usos y resignificaciones en el caso español. En H. Monarca (Coord.), Calidad de la educación en Iberoamérica: Discursos, políticas y prácticas (pp. 1244). Madrid: Dykinson. 
Hernández, C. (2015). Nuevos recursos para la investigación cualitativa: Software gratuito y herramientas colaborativas. Opción, vol. 31, núm. 5, 453-471

Laclau, E. (1996). Emancipación y diferencia. Buenos Aires: Ariel.

López Aguado, M. (2018). La evaluación de la calidad de títulos universitarios. Dificultades percibidas por los responsables de los sistemas de garantía de calidad. Educación $X X I, 21(1)$, pp. 263-284.

Ministros Europeos. (1998). Declaración de la Sorbona. Declaración conjunta para la armonización del diseño del sistema de educación superior europeo. París.

Muñoz-Repiso, M. y Murillo, F.J. (2010). Un balance provisional sobre la calidad en educación: eficacia escolar y mejora de la escuela. Revista Iberoamericana sobre Calidad, Eficacia y Cambio en Educación, 8(2), pp. 177-186.

Paris Communiqué, 25th of may 2018. Conférence ministérielle européenne pour l'enseignement supérieur. Disponible en: http://www.ehea.info/page-ministerialconference-paris-2018. Fecha de acceso: 1 de junio de 2020.

Resolución del Parlamento Europeo, de 20 de abril de 2012, sobre la modernización de los sistemas de educación superior en Europa. (2013/C 258 E/o8). Diario Oficial, serie C, número $258 \mathrm{E}$, de 7 de septiembre de 2013.

Standards and Guidelines for Quality Assurance in the European Higher Education Area (ESG). (2015). Brussels, Belgium.

Yerevan Communiqué of Ministers, 14th and 15th of may 2015. The Bologna Process Revisited: the Future of the European Higher Education Area. Disponible en: http://www.ehea.info/page-ministerial-conference-yerevan-2015. Fecha de acceso: 1 de junio de 2020.

UNESCO. (2015). Educación 2030: Declaración de Incheon y Marco de Acción. París: UNESCO. 